\title{
Modeling and Simulation of a Nonlinear Energy Harvester under Broadband Vibrations
}

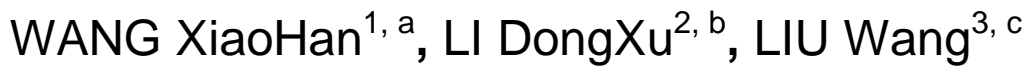 \\ ${ }^{1,2,3}$ College of Aerospace Science and Engineering, National University of Defense Technology, \\ Changsha, Hunan 410073, China. \\ anudt_wangxiaohan@qq.com. \\ bdongxuli@263.net. \\ cwangliu@nudt.edu.cn.
}

Keywords: energy harvesting, nonlinear modeling, broadband vibration

\begin{abstract}
Vibration energy harvesting is a concept to convert the mechanical energy contained in vibrations into useable or storable electrical energy. This article focuses on the influence of nonlinearities upon the output of a piezomagnetoelectric vibration energy harvester (VEH) under broadband vibrations. Nonlinear equations of motion that describe the VEH are given along with numerical simulations. Especially, the relationship between the outputs of the VEH and its nonlinearity is derived, which is meaningful for designing the nonlinearity goal-directed. The numerical analysis presents a comparison between the voltage outputs of a linear $\mathrm{VEH}$ and a nonlinear one under broadband vibrations. Simulation results show that, compared with the linear $\mathrm{VEH}$, the nonlinear VEH presented in this article has a wider working bandwidth and a lower resonant frequency which indicates the nonlinear VEH has a better performance under broadband vibrations.
\end{abstract}

\section{Introduction}

Vibration energy harvesting is a concept to convert the mechanical energy contained in vibrations into useable or storable electrical energy [1-3]. Especially, this idea can be combined with vibration reduction and control purposes, i.e. eliminating undesirable vibrations to generate useful energy.

Linear VEHs, like the piezoelectric cantilever beam, operate based on the basic principle of linear resonance. Efficient energy harvesting can be achieved, only when the base vibration is harmonic with frequencies that equal or close to the beam's modal frequencies. This places critical limitations on its broadband performance. However, most realistic vibrations in the environment have broadband characteristics, namely either the energy is distributed over a wide spectrum of frequencies or the dominant frequency varies with time [4]. Consequently, the narrow spectrum of working frequency critically limits the applicability and usefulness of linear VEHs.

Therefore, no wonder a large portion of the vibration energy harvesting research is currently developed towards designing harvesters capable of harvesting energy from broadband vibrations [4]. One proposed solution is introducing nonlinearities into the VEHs [5-10]. Such VEHs have nonlinear dynamic properties that shall influence the coupling between the base vibration and the harvester and thus broaden its working bandwidth.

This work investigates the influence of the nonlinearity upon the outputs of VEHs under broadband vibration. The designed nonlinear VEH has a configuration of piezomagnetoelectric cantilever beam, of which the nonlinearity comes from nonlinear magnetic forces. Nonlinear equations of motion for the nonlinear VEH are given. In addition, a relationship between the outputs of the VEH and its nonlinearity is theoretically derived, using the d'Alembertl principle. A comparative analysis between the outputs of a linear VEH and a nonlinear VEH is carried out through a numerical approach, which shows a low-frequency-direction moving of resonant frequency and a wider working bandwidth for the nonlinear VEH. Moreover, a further analysis is carried out to define the influence of the base vibration intensity on the extent of nonlinearity. 


\section{Nonlinear VEH modeling}

Designed VEH's nonlinear equations of motion. The designed nonlinear VEH consists of a piezoelectric cantilevered beam with one permanent magnet fixed to its free end, and other two permanent magnets fixed to the base paralleling to the first one at a vertical distance $d$, as shown in Fig. 1(a). The piezoelectric beam is formed by a PZT layer attaching to the root layer, as shown in Fig. 1(b).The voltage output of the PZT layer due to the base vibration is the primary interest in energy harvesting. Facing micro-vibration on spacecraft, choose two type of vibration i.e. 5-200Hz sweep vibration and $5-200 \mathrm{~Hz}$ noise as base vibration. Under this consideration only the first model will be excited, and bending will be the main deformation type.

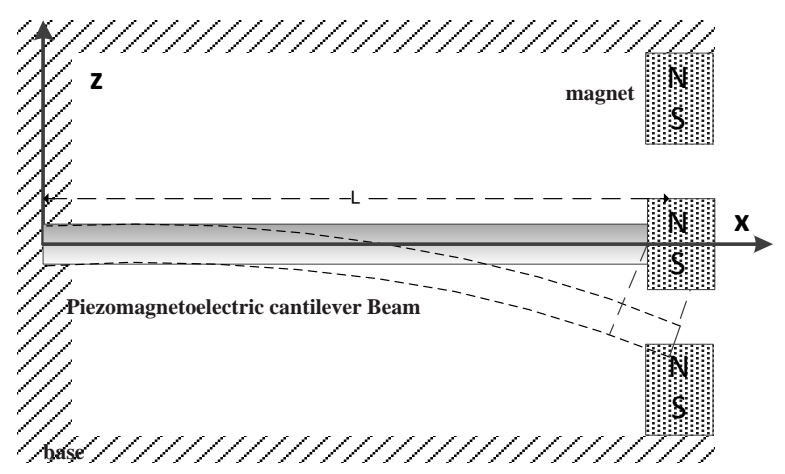

(a)

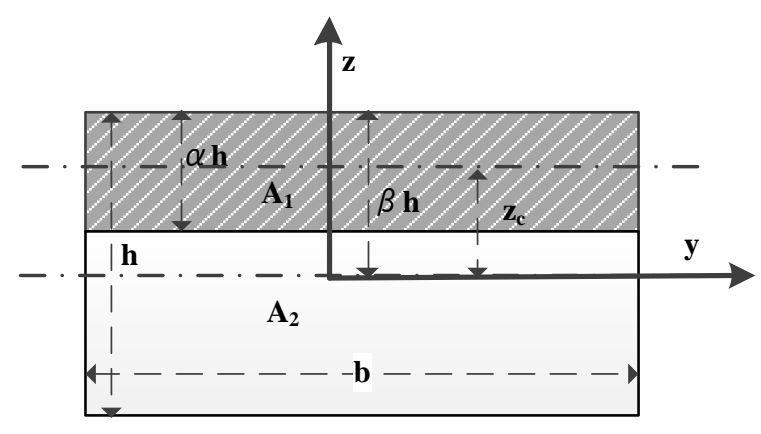

(b)

Fig. 1 Schematic of the vibration energy harvester. (a)front view (b)sectional view

Under the micro-excitation in $Z$ direction, the vibration of ending magnet will occur mainly in $Z$ direction and bending will be the major deformation type of the beam. This can be simplified into a one degree of freedom lumped parameter model as shown in Fig. 2, and the equation of motion can be written as, Eq. 1, which is , obviously, a nonlinear equation owing to the magnetic force.In this model, the displacement $z(t)$ of equivalent mass is assumed to equaling the free end deflection $w(L)$ of cantilever beam when vibrating.

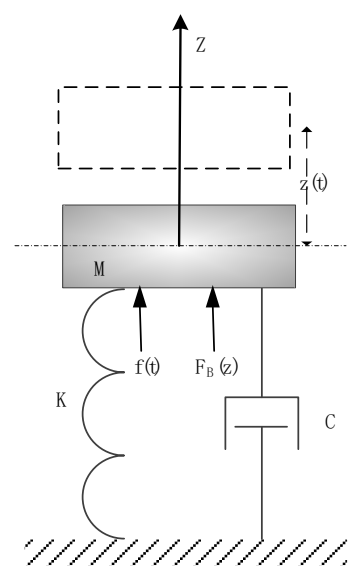

Fig. 2 One degree of freedom lumped parameter model of the designed VEH

$$
M \ddot{z}+C \dot{z}+K z=f(t)+F_{B}(z) .
$$

where: $M=\frac{1}{3} m+m_{0}$, is the equivalent mass, defined by kinetic energy equality when vibrating; $m, m_{0}$ therein, is the mass of cantilever and free-end-magnet, respectively. $K=\frac{3\left(I_{1} e_{1}+I_{2} e_{2}\right)}{L^{3}}$, is the equivalent stiffness, defined by deflection curve of cantilever beam under ending concentrated force; $I_{1}, e_{1}, I_{2}, e_{2}$, therein, are inertia moment and elasticity modulus of PZT layer and root layer, respectively. 
$C=2 \xi \sqrt{M K}$ is the equivalent damping, when assuming damping ratio $\xi=0.01$ by engineering experience.

$f(t)$ is the equivalent force equals to the external force, except magnetic force, acted at the free end of the cantilever beam; and $F_{B}(z)$ is the nonlinear magnetic force of free-end-magnet when vibrating.

When the cantilever beam bending, the average stress along any section equals to the stress at the $\mathrm{z}$ direction middle point of PZT layer $z_{c}$, which can be defined by generalization Hooke law:

$$
T_{c}(x)=e_{1} S_{c}(x)=e_{1} z_{c} \frac{\frac{d^{2} w(x)}{d x^{2}}}{\left[1+\left(\frac{d w(x)}{d x}\right)^{2}\right]^{\frac{3}{2}}} .
$$

where, $z_{c}=\left(\beta-\frac{\alpha}{2}\right) h$ known from Fig. 2; $w(x)$ is the deflection of beam at point $\mathrm{x}$.

Through piezoelectric equation the average electric displacement vector $D(x, y)=d_{31} T_{c}(x)$, and further, the total electric quantity derived as:

$$
Q=\iint_{A} D_{3} d x d y=b z_{c} e_{1} d_{31} \int_{0}^{L_{1}} \frac{\frac{d^{2} w(x)}{d x^{2}}}{\left[1+\left(\frac{d w(x)}{d x}\right)^{2}\right]^{\frac{3}{2}}} d x=\frac{b z_{c} e_{1} d_{31} \theta(x)}{\sqrt{1+\theta(x)^{2}}} \mid \begin{aligned}
& \theta(L) \\
& \theta(0)
\end{aligned} .
$$

where, $\theta(x)=\frac{d w(x)}{d x}$ is the rotation angle at point $x$.

Considering the cantilever boundary condition leading $\theta(0)=0$, meanwhile, under broadband micro-vibration the deformation of the beam meets the micro-deformation assumption leading $\sqrt{1+\theta(L)^{2}} \doteq 1$, simplifying Eq. 3 as:

$$
Q=b z_{c} e_{1} d_{31} \theta(L)
$$

One step further, the instantaneous open circuit voltage can be derived as:

$$
V_{\text {out }}=\frac{Q}{C_{p}}=\frac{b z_{c} e_{1} d_{31} \theta(L)}{C_{p}} .
$$

where, $C_{p}=\frac{\varepsilon_{33} L b}{h_{1}}$, is the equivalent capacitance, and $\varepsilon_{33}$ is the dielectric constant of PZT.

Nonlinear magnetic force modeling. The magnetic force $f_{B}$ equals to the gradient of magnetic energy $E_{B}$ in certain direction, written as:

$$
f_{B}=\nabla E_{B}
$$

When the medium between two near enfiladed magnets is air, the magnetic energy in the medium can be simplified as:

$$
E_{B}=\frac{B_{g}{ }^{2} A_{g} L_{g}}{8 \pi} .
$$


where $A_{g}$ is the magnetic area, $L_{g}$ is the thickness of medium between two magnet, and $B_{g}$ is the magnetic induction intensity at the center point of medium.

For cubic magnet with surface magnetism $B_{r}$ and magnet area size $a \times b$ :

$$
B_{g}=\frac{2 B_{r}}{\pi} \arctan \left(\frac{a b}{2 L_{g} \sqrt{4 L_{g}^{2}+a^{2}+b^{2}}}\right) .
$$

The total magnetic force $F_{B}$ applied on the middle magnet in a group of three cubic magnets shown in Fig. 3, is a function with the distance of middle magnet apart from the middle point. When the middle magnet apart from middle point at distance $z, F_{B}$ derived as:

$$
F_{B}=\frac{a b B_{r}^{2}}{20 \pi^{3}}\left[\left(\arctan \frac{a b}{2(d-z) \sqrt{4(d-z)^{2}+a^{2}+b^{2}}}\right)^{2}-\left(\arctan \frac{a b}{2(d+z) \sqrt{4(d+z)^{2}+a^{2}+b^{2}}}\right)^{2}\right]
$$

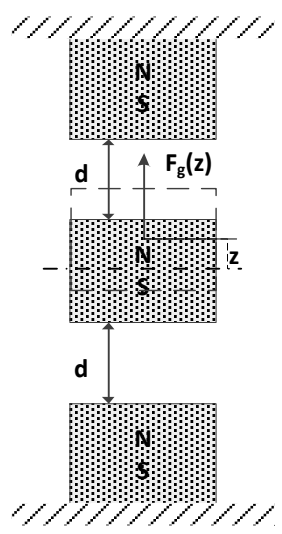

Fig. 3 Three cubic magnets group

Relationship between the outputs and nonlinear force. Using d'Alembertl principle analyze the lumped parameter model of VEH in Fig. 2, the dynamic balance state of the equivalent mass can be equivalent by a static balance under external force $f(t)$, magnetic force $F_{B}$, inertia force $M \ddot{z}$, damping force $C \dot{z}$, and stiffness force $K z$, written as:

$$
f(t)+F_{B}-M \ddot{z}-C \dot{z}-K z=0 .
$$

Then, obviously, there must exist an external force $F_{r}$ leading the equivalent mass to reach the same balance position but in real static state, written as:

$$
F_{r}+F_{B}-K z=0 .
$$

Supposing under broadband micro-vibration only the first model of the cantilever beam will be excited. In this assumption the instantaneous dynamic vibration curve can be simulated, in very high accuracy, by static deflection curve under concentrated force applied on free end. And if the concentrated force is $F_{r}$, the rotation angle at free end is $\theta(L)=\frac{F_{r} L^{2}}{2\left(I_{1} e_{1}+I_{2} e_{2}\right)}$.

Therefore, the relationship between the voltage output of the $\mathrm{VEH}$ and nonlinearity source $F_{B}$ is derived as: 


$$
V_{\text {out }}=\frac{Q}{C_{p}}=\frac{L h_{1} z_{c} e_{1} d_{31}}{2 \varepsilon_{33}\left(I_{1} e_{1}+I_{2} e_{2}\right)}\left(K z-F_{B}\right)
$$

The output of VEH is a function of $z$, shown in Eq. 12. And the main influence factor of its property is the equivalent stiffness and the nonlinear magnetic force $F_{B}$. From Eq. 9, we know $F_{B}$ is a nonlinear function of $z$, which combined with $K z$ determining the nonlinear properties of the designed VEH. Obviously, adding the term $-F_{B}$ will make the whole system softener than befor and meanwhile, $F_{B}$ is the source of nonlinearty. Thus, we say the desined VEH have sofening stiffness nonlinearty.

\section{Numerical simulations}

Numerical simulations for the VEH voltage outputs under two types of broadband excitations, i.e. sweep-frequency excitation and band limited noise excitation, are done on MATLAB platform. The properties of the nonlinear and linear VEHs used for simulation list in Table 1

Table 1 Properties of VEH

\begin{tabular}{|l|l|l|l|}
\hline & PZT layer & Root layer & Free end magnet \\
\hline Material & PZT-5A & $\mathrm{Cu}$ & $\mathrm{N} 35$ \\
\hline Density $\left(\mathrm{kg} / \mathrm{m}^{3}\right)$ & 7450 & 8230 & $10.8 \times 10^{3}$ \\
\hline Length $(\mathrm{mm})$ & 100 & 100 & 10 \\
\hline Width $(\mathrm{mm})$ & 10 & 10 & 10 \\
\hline Thickness $(\mathrm{mm})$ & 2 & 2 & 20 \\
\hline Young's modulus $(\mathrm{pa})$ & $6.098 \times 10^{10}$ & $1.06 \times 10^{11}$ & \\
\hline Piezoelectric coefficient $d_{31}(\mathrm{pC} / \mathrm{N})$ & $-171 \times 10^{-12}$ & & \\
\hline Dielectric constant $\varepsilon_{33}(\mathrm{~F} / \mathrm{m})$ & $1700 \times 8.854 \times 10^{-12}$ & & \\
\hline Surface magnetism $B_{r}(\mathrm{G})$ & & & 4200 \\
\hline
\end{tabular}

5-250 (Hz) sweep-frequency excitation. When 5-250 (Hz) sweep-frequency type input is given, the voltage outputs simlation of nonlinear and linear VEH shows in Fig. 4. In this simulation the excitation amplify changes from $1 \mathrm{~N}$ to $4 \mathrm{~N}$ and frequencies sweep from $5 \mathrm{~Hz}$ to $250 \mathrm{~Hz}$.

Fig. 4 shows, obviously, the resonant frequency of nonlinear case moves towards low frequency, compared with linear case. When the excitatin amplitudes are $1 \mathrm{~N}$ and $2 \mathrm{~N}$, the nonlinearity is not aroused. So the working bandwith is not apparentley widened, and the peak output is lower. However, when the excitation amplitude increased to $3 \mathrm{~N}$ and $4 \mathrm{~N}$ the nonlinearity is aroused, the $3 \mathrm{~dB}$ working bandwith is widened from $2 \mathrm{~Hz}$ to $19 \mathrm{~dB}$ for Fig. 4 (c), from $2 \mathrm{~dB}$ to $25 \mathrm{~dB}$ for Fig. 4 (d) and the peak output also exceeding the linear case.

1-250 (Hz) band limited noise excitation. When 1-250 (Hz) band limited noise type input is given, the voltage outputs of nonlinear and linear VEH shows in Fig. 5.In this simulation the excitation is band limited noise, whose energy destributed uniformly from $1 \mathrm{~Hz}$ to $250 \mathrm{~Hz}$ in frequency domain and the spectral density of excitation increase from $5 \times 10^{-4}$ to $20 \times 10^{-4}\left(\mathrm{~g}^{2} / \mathrm{Hz}\right)$ as Fig. 5 (a) to (d). Similarly, the resonant frequency of nonlinear case also moves towards low frequency, compared with linear case. And when the nonlinearity is not aroused, in Fig. 5 (a) (b), for nonlinear VEH the bandwith is not apparentley widened and the whole voltage output is lower than linear case. However when the nonlinearity is aroused, in Fig. 5 (c) (d), the peak of the output is smoothed but the efective working bandwith is widened from $5 \mathrm{~Hz}$ to $40 \mathrm{~Hz}$ for Fig. 5 (c) and from $5 \mathrm{~Hz}$ to $42 \mathrm{~Hz}$ for Fig. 5 (d), so the total output is increased. 


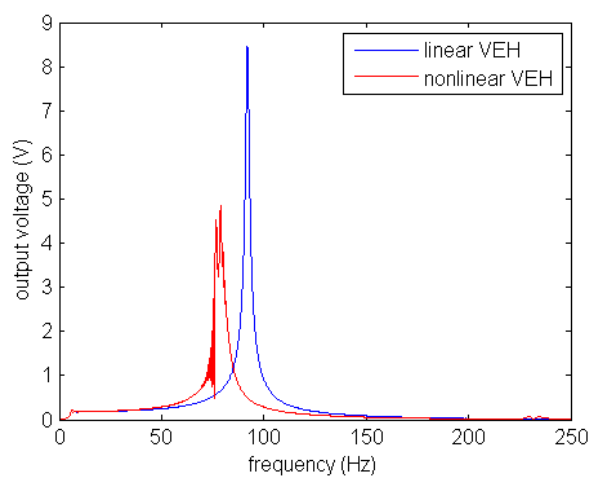

(a)

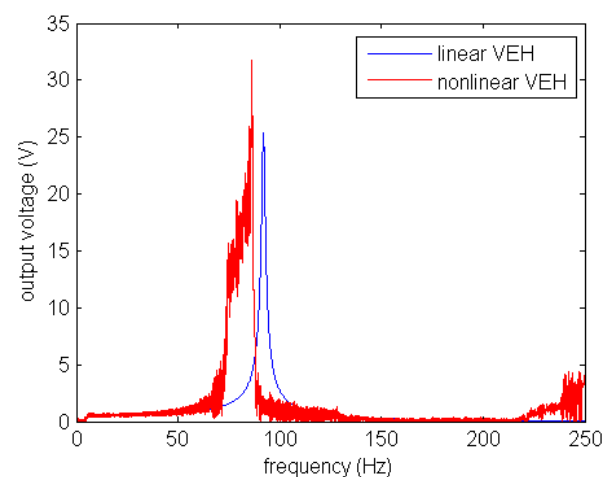

(c)

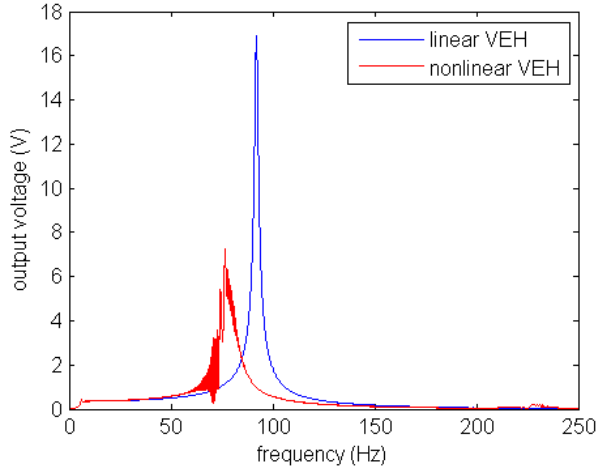

(b)

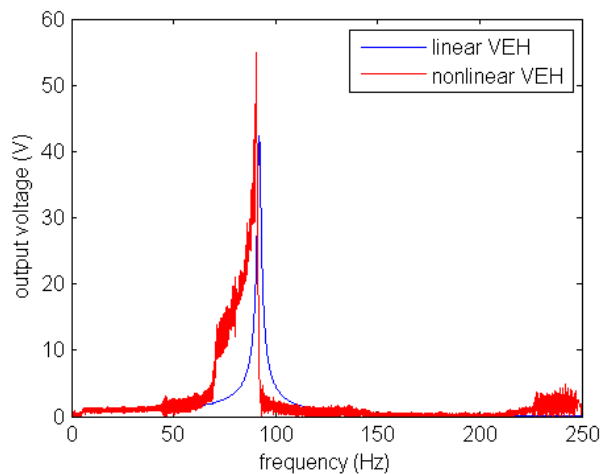

(d)

Fig. 4 Voltage output changing with excitation amplitude (a) 1N, (b) 2N, (c) 3N, (d) 4N

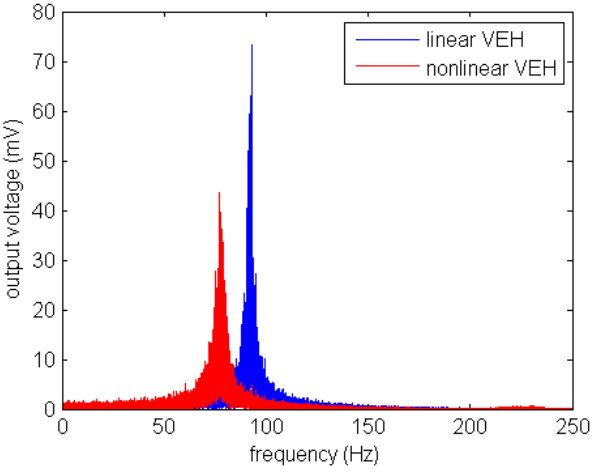

(a)

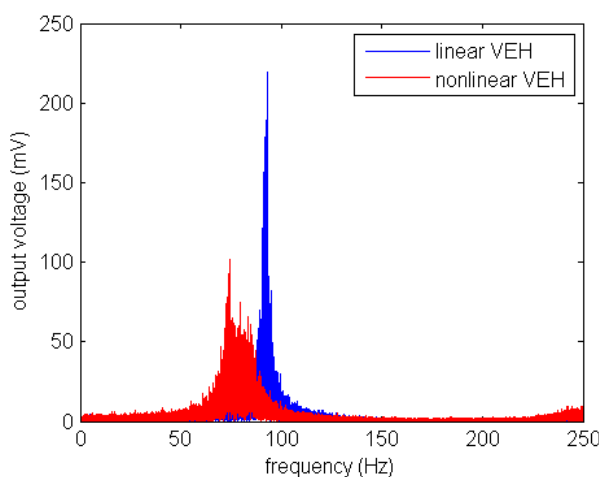

(c)

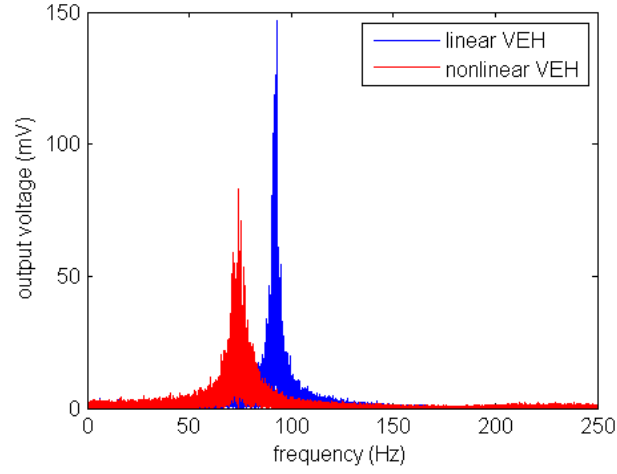

(b)

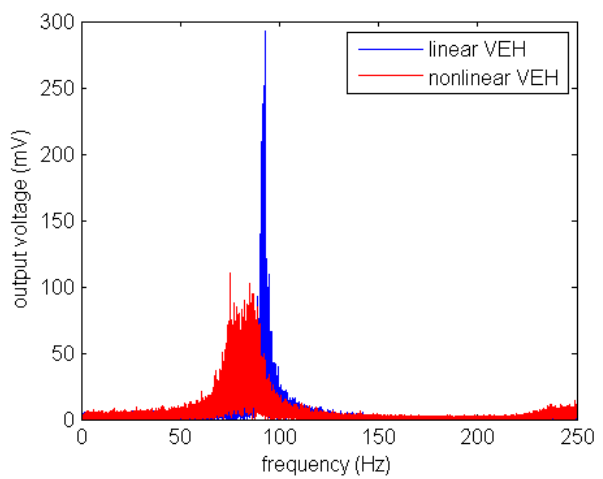

(d)

Fig. 5 Voltage output changing with increasing spectral density of excitation (a) 5 , (b) 10 , (c) 15, (d) $20, \times 10^{-4}\left(\mathrm{~g}^{2} / \mathrm{Hz}\right)$ 


\section{Conclusion}

This article concentrates on exploiting the nonlinearity of VEHs for broadband energy harvesting, via establishing the relationship between the nonlinearity and the system equivalent stiffness. The relationship between the outputs of the VEH and its nonlinearity is theoretically derived as Eq. 12, which explains that the designed nonlinear VEH has a softening stiffness type of nonlinearity. And based on this, any other type of nonlinearity is possible to be designed as long as a proper nonlinear force.

The output of the designed nonlinear $\mathrm{VEH}$ under broadband vibrations is also investigated. Simulation results show that its resonant frequency will move to the low-frequency direction under broadband vibrations, both sweep-frequency type and band limited type. When applying sweep-frequency type excitation, which is strong enough for arousing the nonlinearity of the designed $\mathrm{VEH}$, the output will have a wider bandwidth and a higher peak value compared with the linear response situation. And when the applied excitation is in a band-limited type, also strong enough for arousing the nonlinearity, the output will have a wider bandwidth but a lower peak value compared with the linear response situation. Considering the total output over working bandwidth, the nonlinear VEH is still dominant.

Comprehensively, we think the designed nonlinear VEH has a preferable performance under broadband vibrations, when the amplitude of vibration is strong enough to arouse the nonlinearity characteristic of the VEH.

\section{References}

[1] Sodano, H., Inman, D. J., and Park, G., “A Review of Power Harvesting From Vibration Using Piezoelectric Materials,” Shock Vib. Dig., 36, (2004), 197-205.

[2] Sodano, H., Inman, D. J., and Park, G., "Generation and Storage of Electricity From Power Harvesting Devices,” J. Intell. Mater. Syst. Struct., 16, (2005), 67-75.

[3] Roundy, S., “On the Effectiveness of Vibration-Based Energy Harvesting,”J. Intell. Mater. Struct., 16, (2005) 809-823.

[4] Daqaq MF, Masana R, Erturk A, Dane Quinn DD. “On the Role of Nonlinearities in Vibratory Energy Harvesting: A Critical Review and Discussion.” ASME. Appl. Mech. Rev. 66; (2014).

[5] M.S.M. Soliman, E.M. Abdel-Rahman, E.F. El-Saadany, R.R. Mansour, A wideband vibration-based energy harvester, J. Micromech. Microeng. 18, (2009)

[6] B. Marinkovic, H. Koser, Smart sand-a wide bandwidth vibration energy harvesting platform, Appl. Phys. Lett. 94, (2009).

[7] L. Gammaitoni, I. Neri, H. Vocca, The benefits of noise and nonlinearity: extracting energy from random vibrations, Chem. Phys. 375 (2010) 435-438.

[8] L.V. Blarigan, P. Danzl, J. Moehlis, A broadband vibrational energy harvester, Appl. Phys. Lett. 100, (2012).

[9] F. Giusaa, A. Giuffridaa, C. Trigonaa, B. Andòa, A.R. Bulsarab, S. Baglio, Random mechanical switching harvesting on inductor: a novel approach to collect and store energy from weak random vibrations with zero voltage threshold, Sens. Actuators A 198, (2013), 35-45.

[10] Aline S. De Paula, Daniel J. Inman, Marcelo A. Savi, “Energy harvesting in a nonlinear piezomagnetoelastic beam subjected to random excitation”, Mechanical Systems and Signal Processing, 54-55, (2015), 405-416. 